\title{
An Optimized Cooperative Transmission based on V- BLAST Technique and GA clustering for Wireless Sensor Networks
}

\author{
Mohammad Sadeghian \\ Kerdabadi \\ M.Sc Student \\ Department of electrical and \\ computer engineering \\ Birjand University, Birjand, \\ Iran
}

\author{
Ali Ahmadi \\ M.Sc Student \\ Department of electrical and \\ computer engineering \\ Birjand University, Birjand, Iran
}

\author{
Reza Ghazizadeh \\ Assistant Professor \\ Department of electrical and \\ computer engineering \\ Birjand University, Birjand, Iran
}

\begin{abstract}
Energy saving is an essential issue in the design of a wireless sensor network because the sensor nodes are generally energy-limited. Thus, minimizing and balancing the energy consumption for nodes are becoming important in terms of extending the network lifetime. In this paper, a novel energyefficient cooperative MIMO transmission mechanism based on V-BLAST technique is proposed. Compared with previous presented structures, in proposed scheme the clustering is done based on Genetic Algorithm then V-BLAST technique based cooperative MIMO transmission are used. An energy consumption is developed to investigate the energy saving performance. The performance of suggested protocol is compared with the LEACH and previous work. Simulation results demonstrate that proposed scheme can achieve better network lifetime and decrease the energy consumption of the network.
\end{abstract}

\section{General Terms}

Communication, Sensor Networks, Clustering, Energy Efficient

\section{Keywords}

Wireless sensor network, Algorithm genetic, Cooperative MIMO, V-BLAST technique, Network lifetime

\section{INTRODUCTION}

One of the most important challenge of wireless sensor network is how to prolong its lifetime. Thus a main design requirement of the wireless sensor networks is to reduce the total energy consumption of the sensor nodes. A technique that has been recently introduced in wireless sensor networks focusing on multiple input multiple output (MIMO) mechanism. However, implementing multiple antennas on small sensor nodes seems infeasible. Hence, the concept of the cooperative MIMO is introduced to solve the problem. The use of cooperative communications in WSNs allows for energy saving through spatial diversity gains [1]. In cooperative MIMO, a group of sensor nodes that is called cluster are selected to cooperatively transmit or receive, thus a method to improve the lifetime is to reduce the number of transmissions by clustering the nodes [2]. In this approach each group of sensors has a cluster head node that collects and aggregates data received from its cluster members and thus decrease the number of relayed packets. During the last few years, many clustering algorithms have been proposed in the literature for WSNs which can be classified based on two criteria [3]: (1) the parameters used for electing $\mathrm{CHs}$ and (2) the execution nature of the clustering algorithm (probabilistic or iterative). Low Energy Adaptive Clustering Hierarchy $(\mathrm{LEACH})$ is one of the most popular energy efficient clustering algorithms for WSNs that forms clusters based on the received signal strength and uses these local cluster heads as routers to the BS [2]. LEACH protocol utilizes randomized rotation of cluster heads to evenly distribute the energy load among the sensors in the network. But random selection of cluster heads may lead to improper distribution in the network. This means that in a part of the network under coverage, two or more adjacent cluster heads are placed close together, whereas in any other region there would not be a cluster head. So, LEACH does not guarantee that the positions evenly placed across the network and the desired number of cluster heads is selected. Its proposer later improved it, proposing the LEACH-C [4]. The main improvement was that during the clustering nodes no longer compete for cluster heads, but each node sends own information about its current location and residual energy level to the BS then the BS node determines the location of cluster heads according to their energy, location and cycle. In addition to determining good clusters, the BS needs to ensure that the energy load is evenly distributed among all the nodes. The optimum selection of the cluster heads with high energy, their number and cluster members are NP-hard problem. Hence evolutionary algorithms are generally more suitable to solve these problems because they are population based stochastic approaches that can avoid being trapped in a local optimum and can often find global optimum solution [5]. Introduction of artificial intelligence in $\mathrm{CH}$ selection shows an improvement of network lifetime compared to classical random selection method. Sajid Hussain and et al [6] successfully utilized a genetic algorithm approach to clustering the nodes. In [7] the authors used PSO to equalize the number of nodes and candidate cluster heads in each cluster in order to minimize the energy expended by the nodes while maximizing the data transmission. In [8], Abdul latiff and et al proposed a centralized cluster based protocol to expend the sensor network lifetime by using PSO and GA algorithms that can minimize the maximum intra cluster distance between cluster head and the cluster members and the optimization of energy management of the network so, they proved that both PSO and GA can be give optimal clustering compared with LEACH and LEACH-C algorithm. Furthermore, PSO algorithm gives a higher network lifetime compared with GA. 
In [5] author utilized a clustering algorithm based on Honey Bee Mating optimization (HBMO) algorithm to optimize clustering process by considering the energy and consumption costs and other factors. Cupta's literature shows an improvement of network lifetime over LEACH protocol but it only selects one cluster head $(\mathrm{CH})$ in each round. In this method, during each period, the sensor that has the most chance is selected as cluster head [9]. Three fuzzy variables are used to calculate the chance including: the number of neighbors of the nodes, residual energy of the nodes and centrally [9]. Siew and et al described the use of fuzzy logic to select suitable $\mathrm{CH}$ among sensor nodes [10]. [11] proposed a clustering algorithm based on Imperialist competitive algorithm (ICA) that, this method acts on one of the clusters in the network to choose the best sensor in the cluster as a cluster head to perform this action, the cluster is divided into several sub clusters, each of which has a cluster head. These cluster heads using assimilation policies try to attract the regular nodes to themselves and using Imperialistic compete with each other until one of these cluster heads is selected as the final cluster head. After this stage, the algorithm work ends. The other ways to improve wireless sensor networks based on cooperative MIMO is data transmission structure kinds. Many of these structures are based on V-BLAST technique. In [12] authors propose a receiver side vertical Bell Laboratories layered Space time (V-BLAST) MIMO processing communication architecture where the transmitter side jointly encoding is indispensable. Authors in [13], propose a delay and channel estimation scheme without transmission synchronization for decoding in such cooperative MIMO scheme. In [4], an energy efficient cooperative MMO transmission scheme based on V-BLAST technique is proposed which not require transmitter side cooperation. Ding .Jie and et al instead of using cluster members as cooperative nodes, multiple cluster nodes cooperative to form virtual array so that MIMO transmission can be implemented. According to the MIMO techniques used in this architecture. V-BLAST based cluster head cluster heads cooperative transmission (VCHCT) are developed [14].

In this paper, the previous work in [14] is extended as follows: In the proposed mechanism after forming clusters by GA algorithm in each rounds, the number of nodes in each clusters are selected as cooperative nodes. These is nodes are called VBN (V-BLAST nodes). Now, after a cluster head receives data packets from its cluster members, it will perform data aggregation and then distribute the fused data to preselected VBN nodes. In the next step, VBN nodes simultaneously transmit the data packets to the BS.

The organization of the rest of this paper is as follows:

Section 2 present the system model and the proposed scheme. In section 3 the energy consumption model for collaborative communication is presented. In section 4 simulation results in Matlab software are given and analyzed and finally in section 5 is the conclusion.

\subsection{Overview of Genetic Algorithm}

Genetic Algorithm (GA) is an adaptive methods that is generally employed to solve search and optimization problems.GA maintains a population consists of a collection of strings called chromosomes and those repeatedly modifies according to selection, mutation, crossover and replacement methods. Each chromosome represents different points in the search space and has a measure of goodness called fitness or objective function. Each chromosome consists of a sequence of bits in which every bit is represented as a sensor node that can be 0 or 1 . A " 1 " means that corresponding sensor is a cluster head and a " 0 " means that it is only a member of a cluster. We utilize GA to determine the number of cluster heads and choosing the best ones. The mutation methods is applied to each bit of an individual with a probability of mutation rate. Next step in creating a new population is the crossover. The crossover operation takes place between two consecutive individuals with probability specified by crossover rate. These two individuals exchange portions that are separated by the crossover point. The selection process means to extract a subset of the chromosomes from the current population will mate (crossover) to create new chromosomes. These new chromosomes joint to the existing population. This combined population will be the basis for the next selection. The chromosome with better fitness value have better chances of selection. This procedure is repeated until convergence is reached or until a maximum number of generations are achieved. Figure 1 indicates diagram of the GA algorithm. We explain the parameters of our GA fitness function in brief. We consider the number of cluster heads as a main parameter for selecting the cluster heads. Other mains parameters are residual energy of the all nodes and distance between cluster members and their cluster head. The lower the communication distance the less energy will be consume during transmission. As cluster heads receive more data and transmit them to sink, so nodes with a more residual energy are better choices to become cluster heads. Therefore we use the same fitness function as stated in [8]. Eq.1 shows cost function.

$$
\begin{gathered}
\text { Fitness }=(1-\alpha) \times\left[\operatorname{SUM}\left(\mathrm{E}\left(\mathrm{n}_{\mathrm{I}}\right)\right) / \operatorname{SUM}\left(\mathrm{E}\left(\mathrm{CH}_{\mathrm{k}}\right)\right)\right]+ \\
\alpha \times\left[\max \left\{\operatorname{SUM}\left(\mathrm{d}\left(\mathrm{n}_{\mathrm{i}}, \mathrm{CH}_{\mathrm{k}}\right)\right) /\left|\mathrm{C}_{\mathrm{k}}\right|\right\}\right]
\end{gathered}
$$

Where first term represent the ratio of initial energy of all nodes $\mathrm{n}_{\mathrm{i}}, \mathrm{i}=1,2, \ldots, \mathrm{N}$ in the network with the total current energy of the cluster head candidates in the current round and second term represents maximum average Euclidean distances from all cluster nodes toward their cluster head and $\left|C_{k}\right|$ is the number of nodes that belong to cluster $\mathrm{C}_{\mathrm{k}}, \mathrm{k}=1,2, \ldots, \mathrm{K}$. The $\alpha$ used to weigh the portion of each of the sub -objectives. The BS uses the optimization algorithm to specify the best $\mathrm{K}$ cluster heads that can minimize the cost function defined above. 


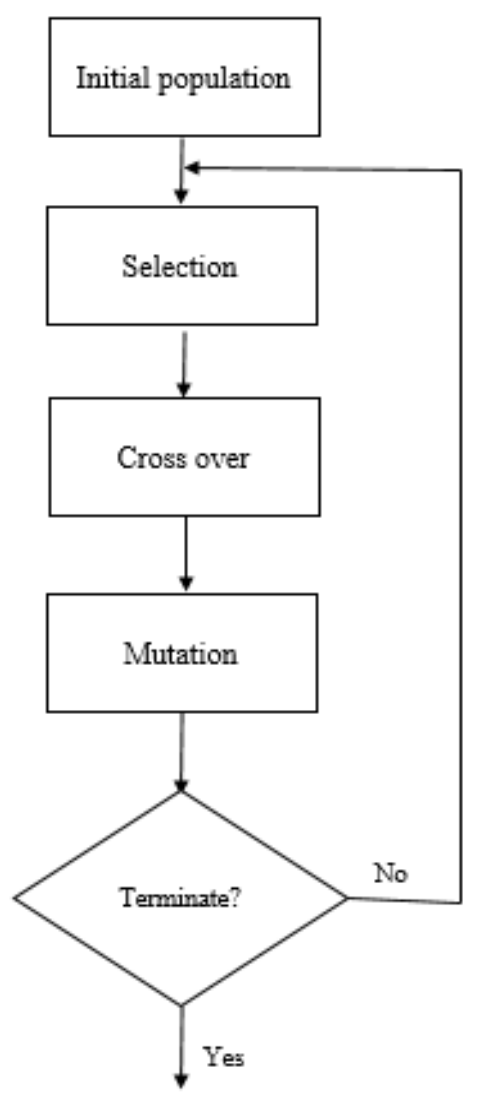

Fig 1: Schematic diagram of the GA algorithm

\section{SYSTEM MODEL AND STRUCTURE}

Assume a sensor network with the following properties. As shown in Figure 2:

- The sensor network consist of $\mathrm{N}$ sensor nodes distributed over on area $\mathrm{M} \times \mathrm{M}$.

-The nodes power control capabilities to vary their transmission power.

-Data fusion is used to reduce the total data message sent.

-Fixed rate BPSK is used in analysis.

In the first step all the sensor nodes will organized themselves into $\mathrm{K}$ clusters based on the Genetic Algorithm. After selecting the cluster heads and formation of the clusters, number of nodes in each cluster are chosen as V-BLAST nodes (VBN) for MIMO transmission, the VBN nodes are chosen by a threshold $\delta$ where $\delta=\frac{E_{\text {remain }}}{\mathrm{d}_{\text {htovn }}+\mathrm{d}_{\mathrm{vntos}}}$ , $\mathrm{E}_{\text {remain }}$ is the remaining energy of each node. $\mathrm{d}_{\text {htovn }}$ and $d_{v n t o s}$ are distance between the cluster head and VBN nodes and distance between VBN nodes and the sink respectively. Assume $\mathrm{N}_{t}$ VBN nodes should be selected in one clusters and all the nodes except the header node are possible candidates for VBN nodes.

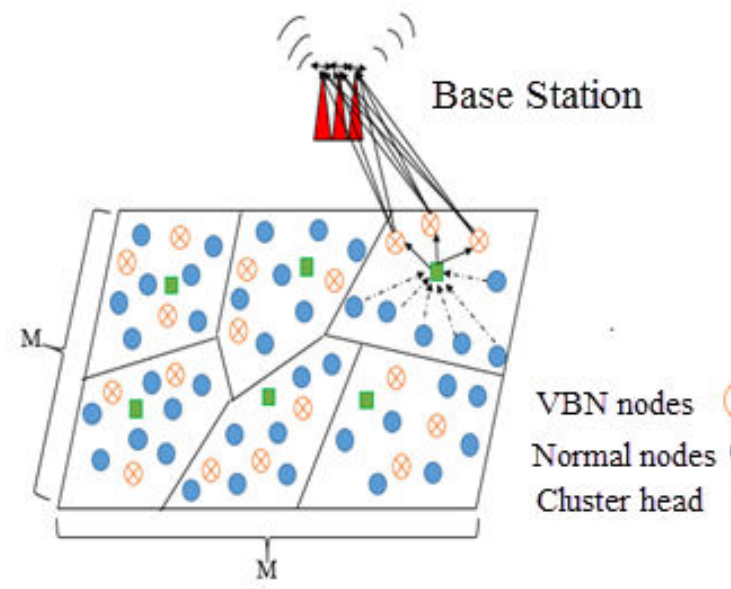

Fig 2: System Model

The $\mathrm{N}_{\mathrm{t}}$ nodes with the maximum value of $\delta$ as the VBN nodes are chosen in the each cluster. In the next step cluster members transmit $\mathrm{L}$ bits data to the cluster heads according to TDMA schedule. After the cluster head receives data packets from its cluster members will first aggregates the received data, then split the fused data into $\mathrm{N}_{\mathrm{t}}$ parts and distribute the split data to the $\mathrm{N}_{\mathrm{t}}$ VBN nodes simultaneously. Finally the sink will receive all data with $\mathrm{N}_{\mathrm{r}}$ antennas and the decorrelating decision feedback detector (D-DFD) [15],[16] are used as the V-BLAST detector at the sink.

\section{ROPOSED SCHEME ENERGY CONSUMPTION MODEL}

Energy consumption of our proposed mechanism can be divided into three main parts: energy consumed by cooperative MIMO transmission and by the normal and VBN nodes in the cluster. To compute the energy of the whole network, first, the energy consumption of transmitting or receiving one bit is calculated. As described in [17] the total power along a signal path can be divided into two major components: the power consumption of all the power amplifiers $\left(\mathrm{P}_{\mathrm{PA}}\right)$ and power consumption of all circuit blocks $\left(\mathrm{P}_{\mathrm{c}}\right)$. Therefore, the transmitting energy consumption of one bit is defined as:

$\mathrm{E}_{\mathrm{bt}}=\frac{\mathrm{P}_{\mathrm{PA}}+\mathrm{P}_{\mathrm{C}}}{\mathrm{R}_{\mathrm{b}}}$

The total power consumption of the power amplifiers can be obtained as: $\mathrm{P}_{\mathrm{PA}}=(1+\alpha) \mathrm{P}_{\text {out }}$ where $\mathrm{P}_{\text {out }}$ is the transmit power, $\alpha=\frac{\xi}{\eta}-1$ with $\prod$ denotes the peak to average ratio that depends on the modulation scheme and the constellation size and $\xi$ denotes drain efficiency of the RF power amplifier. For $\mathrm{P}_{\text {out }}$ two models are defined based on the transmission distance. If the transmission distance is above a threshold free space model is considered otherwise two-ray ground reflection model is assumed. Thus:

$P_{\text {out }}= \begin{cases}E_{b} R_{b} \frac{(4 \pi)^{2} M_{l} N_{f}}{G_{t} G_{r} \lambda^{2}} d^{2} & d \leq d_{0} \\ E_{b} R_{b} \frac{M_{l} N_{f}}{G_{t} G_{r} h_{t}^{2} h_{r}^{2}} d^{4} & d>d_{0}\end{cases}$

where $d$ is the transmission distance. $M_{l}$ is the link margin compensating the hardware process variations and other additive background noise or interference, $G_{t}$ and $G_{r}$ are the transmitter and receiver antenna gains, respectively, $\mathrm{N}_{\mathrm{f}}$ is the 
receiver noise figure defined as $\mathrm{N}_{\mathrm{f}}=\frac{\mathrm{N}_{\mathrm{r}}}{\mathrm{N}_{0}}$ where $\mathrm{N}_{0}$ is the singlesided thermal noise power spectral density at room temperature and $\mathrm{N}_{\mathrm{r}}$ is the PSD of the total effective noise at the receiver input. $\lambda$ is the carrier wavelength. $h_{t}$ and $h_{r}$ are the heights of transmitter and receiver antenna, $R_{b}$ is the system bit rate [14]. $E_{b}$ is the required average energy per bit at the receiver for a given BER requirement $\mathrm{P}_{\mathrm{b}}$, To estimate the values of the circuit power consumption at transmitter side, $\mathrm{P}_{\mathrm{ct}}$ and at the receiver side, $\mathrm{P}_{\mathrm{cr}}$, therefore :

$$
\begin{gathered}
\mathrm{P}_{\mathrm{ct}}=\mathrm{P}_{\mathrm{DAC}}+\mathrm{P}_{\mathrm{mix}}+\mathrm{P}_{\text {filt }}+\mathrm{P}_{\mathrm{syn}} \\
\mathrm{P}_{\mathrm{cr}}=\mathrm{P}_{\mathrm{LNA}}+\mathrm{P}_{\mathrm{ADC}}+\mathrm{P}_{\mathrm{mix}}+\mathrm{P}_{\text {filr }}+\mathrm{P}_{\text {syn }}+\mathrm{P}_{\mathrm{IFA}}
\end{gathered}
$$

$\mathrm{P}_{\mathrm{DAC}}, \mathrm{P}_{\text {mix }}, \mathrm{P}_{\text {filt }}, \mathrm{P}_{\text {syn }}, \mathrm{P}_{\text {LNA }}, \mathrm{P}_{\mathrm{ADC}}, \mathrm{P}_{\text {filr }}, \mathrm{P}_{\text {syn }}$ and $\mathrm{P}_{\text {IFA }}$ are the power consumption values for the $\mathrm{D} / \mathrm{A}$ convertor, the mixer, the active filters at the transmitter side, The frequency synthesizer, the low noise amplifier, A/D convertor, the active filters at the receiver side and the intermediate frequency amplifier, respectively. Assume an AWGN channel and free space model for intra cluster communications and a Rayleigh fading channel and a two-ray ground reflection model for cooperative communications.

\subsection{Energy consumption for intra cluster communication}

The energy consumption for transmitting one bit from normal node to the cluster head can be written as:

$$
\mathrm{E}_{\mathrm{bt}}^{\text {ntoCH }}=\left(\mathrm{n}_{\mathrm{c}}-1\right)(1+\alpha) \mathrm{E}_{\mathrm{b}}^{\mathrm{ntoCH}} \frac{(4 \pi)^{2} \mathrm{M}_{\mathrm{l}} \mathrm{N}_{\mathrm{f}}}{\mathrm{G}_{\mathrm{t}} \mathrm{G}_{\mathrm{r}} \lambda^{2}} \mathrm{~d}_{\text {ntoCH }}^{2}
$$

where $\mathrm{E}_{\mathrm{bt}}^{\mathrm{ntoCH}}$ is energy consumption for transmitting one in the cluster, $d_{n t o C H}$ is the average distance from cluster member to the cluster head which can be approximated as $\left[\mathrm{d}_{\text {ntoCH }}^{2}\right]=\frac{\mathrm{M}^{2}}{2 \pi \mathrm{K}_{\mathrm{c}}}$ where $\mathrm{K}_{\mathrm{c}}$ is number of clusters [18]. $\mathrm{n}_{\mathrm{c}}$ is the number of the nodes in each cluster, $E_{b}^{\text {ntoCH }}$ is the required energy per bit for a given BER requirement which can be expressed by $\mathrm{N}_{0}\left(\mathrm{Q}^{-1}\left(\mathrm{P}_{\mathrm{b}}\right)\right)^{2} / 2$ where $\mathrm{Q}^{-1}$ is inverse $\mathrm{Q}$-function. Thus the $\mathrm{E}_{\mathrm{bt}}^{\mathrm{ntoCH}}$ can be expressed as follows:

$$
E_{b t}^{n t o C H}=\left(n_{c}-1\right)(1+\alpha) E_{b}^{n t o C H} \frac{(4 \pi)^{2} M_{l} N_{f}}{G_{t} G_{r} \lambda^{2}} \frac{M^{2}}{2 \pi K_{c}}
$$

The circuit blocks energy consumption of intra cluster communication can be written as:

$E_{c}^{n t o C H}=\frac{\left(n_{c}-1\right) p_{c t}}{R_{b}}+\frac{p_{c r}}{R_{b}}$

Assume $E_{\mathrm{DA}}$ is the energy consumption for data fusion per bit in the cluster by the cluster head in each round [18]. Then the energy consumption of data fusion is given by:

$$
\mathrm{E}_{\mathrm{fus}}=\mathrm{E}_{\mathrm{DA}} \mathrm{L}\left(\mathrm{n}_{\mathrm{c}}-1\right)
$$

and data length after data fusion by cluster head is expressed as

$$
L_{\text {agg }}=\frac{L\left(n_{c}-1\right)}{f_{a g g}\left(n_{c}-1\right)-f_{a g g}+1}
$$

The energy consumption for transmission aggregated data from cluster head to VBN nodes can be calculate the same as above thus is assumed $\mathrm{E}_{\mathrm{b}}^{\text {ntoCH }}=\mathrm{E}_{\mathrm{b}}^{\text {CHtovn }}$

$$
\mathrm{E}_{\mathrm{bt}}^{\text {CHtovn }}=(1+\alpha) \mathrm{E}_{\mathrm{b}}^{\text {CHtovn }} \frac{(4 \pi)^{2} \mathrm{M}_{\mathrm{l}} \mathrm{N}_{\mathrm{f}}}{\mathrm{G}_{\mathrm{t}} \mathrm{G}_{\mathrm{r}} \lambda^{2}}\left(\mathrm{~d}_{\text {CHtovn }}\right)^{2}
$$

and circuit blocks energy consumption is:

$\mathrm{E}_{\mathrm{C}}^{\text {CHtovn }}=\frac{\mathrm{p}_{\mathrm{ct}}}{\mathrm{bB}}+\frac{\mathrm{N}_{\mathrm{r}} \mathrm{p}_{\mathrm{cr}}}{\mathrm{bB}}$

\subsection{Cooperative MIMO Transmission Energy Consumption Model}

As in [20], the average probability of joint symbol errors of the V-BLAST receiver can be defined as:

$\mathrm{P}_{\mathrm{s}} \approx 1-\prod_{\mathrm{k}=1}^{\mathrm{N}_{\mathrm{t}}}\left(1-\mathrm{p}_{\mathrm{k}}\right)$

$p_{k}=4\left(\frac{\sqrt{2}-1}{\sqrt{2}}\right)\left(\frac{1-\tau}{2}\right)^{k} \sum_{i=0}^{k-1}\left(\begin{array}{c}k-1+i \\ i\end{array}\right)\left(\frac{1+\tau}{2}\right)^{i}$

Where $\mathrm{k}=1, \ldots, \mathrm{N}_{\mathrm{t}}, \tau=\frac{\gamma}{\gamma+1}, \gamma=\frac{\mathrm{E}_{\mathrm{b}}^{\text {mimo }}}{\mathrm{N}_{0}}, \mathrm{E}_{\mathrm{b}}^{\text {mimo }}$ is required

average energy per bit for a given BER, $\overline{\mathrm{P}_{\mathrm{b}}}$,As in [4]. The given average BER of the V-BLAST receiver can be estimated as:

$\overline{\mathrm{P}_{\mathrm{b}}} \approx \mathrm{P}_{\mathrm{S}}\left(\frac{\mathrm{N}_{\mathrm{t}} \mathrm{b}+8}{8 \mathrm{~N}_{\mathrm{t}} \mathrm{b}}\right)$

Thus the energy consumption of transmitting one bit for $\mathrm{V}$ BLAST based MIMO technique is:

$$
\begin{array}{r}
E_{b t}^{\text {mimo }}=\frac{P_{P A}+N_{t} P_{c t}+N_{r} P_{c r}}{R_{b}}= \\
(1+\alpha) E_{b}^{\text {mimo }} \frac{M_{l} N_{f}}{G_{t} G_{r} h_{r}^{2} h_{t}^{2}} d_{v n t o s}^{4}+\frac{N_{t} P_{c t}+N_{r} P_{c r}}{R_{b}}
\end{array}
$$

Where $d_{v n t o s}$ is the distance from the VBN nodes to the sink. Therefore, the total energy consumption for the whole network can be obtain as:

$$
\begin{gathered}
\mathrm{E}_{\text {total }}=\sum_{\mathrm{i}=1}^{\mathrm{k}_{\mathrm{c}}}\left(\mathrm{L}_{\mathrm{agg}} \mathrm{E}_{\mathrm{bt}}^{\text {mimo }}+\mathrm{LE}_{\mathrm{bt}}^{\mathrm{ntoCH}}+\mathrm{LE}_{\mathrm{c}}^{\mathrm{ntoCH}}+\right. \\
\left.\mathrm{L}_{\mathrm{agg}} \mathrm{E}_{\mathrm{bt}}^{\mathrm{CHtovn}}+\mathrm{L}_{\mathrm{agg}} \mathrm{E}_{\mathrm{c}}^{\mathrm{CHtovn}}+\mathrm{E}_{\mathrm{fus}}\right)
\end{gathered}
$$

\section{SIMULATION RESULTS}

In this section, simulations are carried out on the basis of the proposed protocol. The energy consumption model used in [2],[21] is considered for LEACH algorithm. The list of the used simulation parameters and their values are shown in Tables.1, 2 and 3.

Table 1.The Used Parameters in GA Algorithm

\begin{tabular}{|c|c|}
\hline parameters & Value \\
\hline $\mathrm{N}$ & 100 \\
\hline $\begin{array}{c}\text { Initial energy of } \\
\text { each node }\end{array}$ & $5 \mathrm{j}$ \\
\hline Packet Size & $4000 \mathrm{bit}$ \\
\hline Population Size & 100 \\
\hline
\end{tabular}




\begin{tabular}{|c|c|}
\hline Crossover rate & 0.9 \\
\hline Cross over Type & Single point \\
\hline Mutation rate & 0.1 \\
\hline $\begin{array}{c}\text { Number } \\
\text { generation }\end{array}$ & 500 \\
\hline Selection type & Roulette Wheel \\
\hline
\end{tabular}

Table 2. The LEACH Algorithm Parameters

\begin{tabular}{|c|c|}
\hline parameters & Value \\
\hline BS location & $\begin{array}{c}\mathrm{x}=100 \mathrm{~m}, \mathrm{y}=150 \\
\mathrm{~m}\end{array}$ \\
\hline $\begin{array}{c}\text { Number of } \\
\text { nodes(n) }\end{array}$ & 100 \\
\hline Network Size & $100 \mathrm{~m} \mathrm{x} 100 \mathrm{~m}$ \\
\hline Packet size(k) & $5 \mathrm{j}$ \\
\hline $\mathrm{E}_{\text {elec }}$ & $50 \mathrm{nj}$ \\
\hline $\mathrm{E}_{\mathrm{DA}}$ & $50 \mathrm{nj}$ \\
\hline $\begin{array}{c}\text { Amplifier } \\
\text { energy free } \\
\text { space }\left(\varepsilon_{f s}\right)\end{array}$ & $10 \mathrm{Pj} / \mathrm{bit} / \mathrm{m}^{2}$ \\
\hline $\begin{array}{c}\text { Amplifier } \\
\text { energy } \\
\text { multipath }\left(\varepsilon_{m p}\right)\end{array}$ & $0.013 \mathrm{Pj} / \mathrm{bit} / \mathrm{m}^{4}$ \\
\hline \multicolumn{2}{|c}{} \\
\hline
\end{tabular}

Table 3. The System Communication Parameters

\begin{tabular}{|c|c|c|}
\hline $\begin{array}{c}\text { Nodes } \\
\text { number=100 }\end{array}$ & $\begin{array}{c}\text { Sensor } \\
\text { area }=100 \mathrm{~m} \mathrm{x} \\
100 \mathrm{~m}\end{array}$ & $\begin{array}{c}\text { Nodes initial } \\
\text { energy }=5 \mathrm{j}\end{array}$ \\
\hline$\alpha=0.47$ & $\begin{array}{c}\mathrm{P}_{\text {filt }}=\mathrm{P}_{\text {fill }}=2.5 \\
\text { mw }\end{array}$ & $\mathrm{f}_{\mathrm{c}}=2.5 \mathrm{KHZ}$ \\
\hline$\lambda=0.12 \mathrm{~m}$ & $\mathrm{P}_{\mathrm{LNA}}=20 \mathrm{mw}$ & $\mathrm{P}_{\mathrm{b}}=0.003 \mathrm{mw}$ \\
\hline $\mathrm{G}_{\mathrm{t}} \mathrm{G}_{\mathrm{r}}=5 \mathrm{dBi}$ & $\mathrm{P}_{\mathrm{syn}}=50 \mathrm{mw}$ & $\mathrm{M}_{\mathrm{l}}=40 \mathrm{~dB}$ \\
\hline $\mathrm{N}_{\mathrm{f}}=10 \mathrm{~dB}$ & $\begin{array}{c}\mathrm{N}_{0} / 2=-135 \\
m / \mathrm{HZ}\end{array}$ & $\mathrm{P}_{\mathrm{IFA}}=20 \mathrm{mw}$ \\
\hline $\mathrm{P}_{\mathrm{mix}}=30.3 \mathrm{~m}$ & $\mathrm{~B}=10 \mathrm{KHz}$ & $\mathrm{f}_{\mathrm{agg}}=0.7$ \\
\hline $\mathrm{R}=0.75$ & $\mathrm{~L}=4000 \mathrm{bit}$ & $\mathrm{b}=1$ \\
\hline $\mathrm{h}_{\mathrm{t}}=\mathrm{h}_{\mathrm{r}}=1 \mathrm{~m}$ & $\mathrm{P}_{\mathrm{ADC}}=20 \mathrm{mw}$ & $\mathrm{E}_{\mathrm{DA}}=50 \mathrm{nj}$ \\
\hline
\end{tabular}

The proposed scheme is compared with LEACH and Previous proposed architecture in [14]. The difference between the proposed protocol and the scheme in [14] can be described as follows. In previous approach. First, LEACH is used to select suitable cluster head among sensor nodes in the network, then the V-BLAST technique based cooperative MIMO transmission are used, but in proposed scheme the clustering is done based on Genetic Algorithm. The selection criterion for VBN nodes in both methods is same. Figure 3 indicate the results of sensor network clustering by the Genetic Algorithm during the twentieth round.

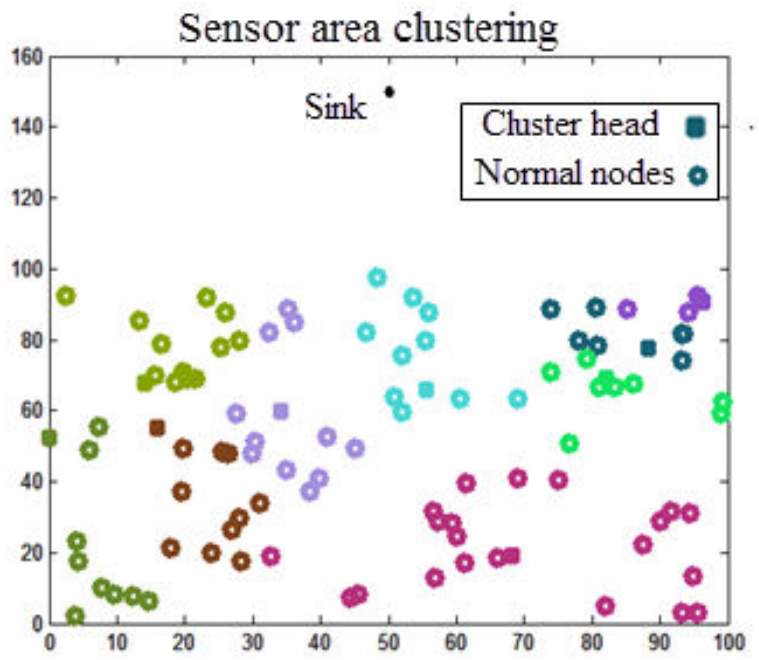

Fig. 3. Sensor network clustering by Genetic Algorithm

Figure 4 shows the amount of total residual energy in network over round for LEACH, proposed scheme and previous presented scheme. It is obvious that the proposed system is more energy efficient than two other methods. As can be seen from this figure, the proposed scheme offers $5 \% 10 \%$ of energy saving compared to previous presented architecture and provides $10^{\mathrm{N}} 15 \%$ of energy saving relative to that of the LEACH.

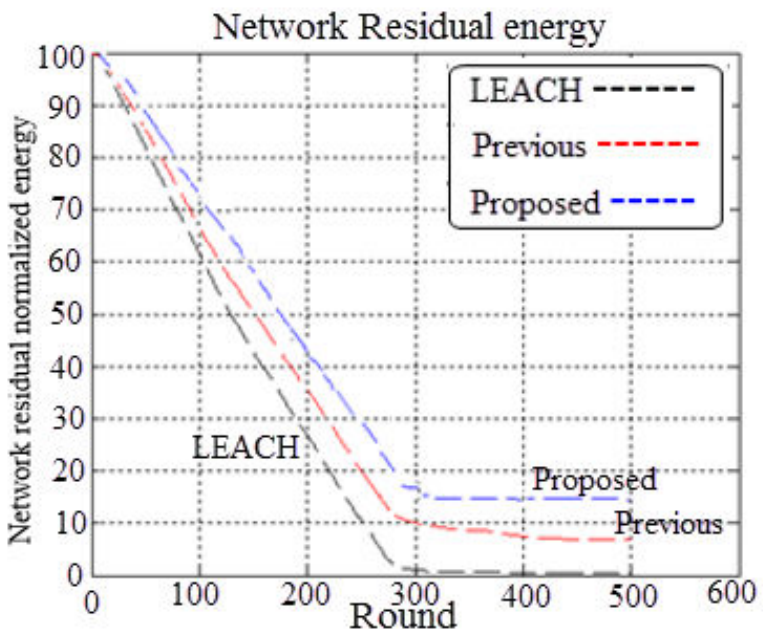

Fig 4. Network Residual Energy vs. simulation round

Figure 5 represent the systems lifetime defined by the number of dead nodes during different rounds. The figure compares the lifetime of the proposed cooperative V-BLAST MIMO scheme with LEACH and previous method. It can be observed that the proposed protocol has considerably less number of dead nodes in the next rounds compared to other methods. 


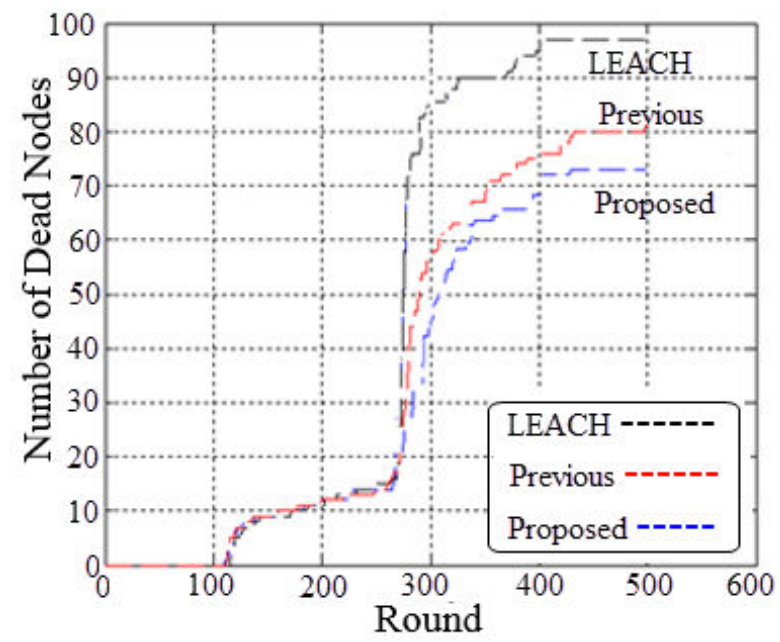

Fig 5.Number of Dead Nodes vs. simulation round

As can be seen the performance of proposed V-BLAST based cooperative MIMO scheme is improved significantly compared with the other methods. Because the performance of the clustering based on genetic algorithm is better than LEACH algorithm and the Genetic Algorithm in cluster head selection shows an improvement of network lifetime compared classical random selection method.

\section{CONCLUSION}

In this paper, a cooperative MIMO transmission scheme for WSNs based on V-BLAST receiver processing to reduce total energy consumption of the network and increase the network lifetime is proposed. In this scheme, the presented approach in [14] are extended by improving the Clustering method. In fact, the LEACH clustering algorithm is replaced with clustering based on Genetic Algorithm. The Simulation results illustrate that firstly, the proposed algorithm can decrease the energy consumption of the network and prolong the network lifetime .Secondly, the proposed cluster head selection scheme makes better selections of cluster heads to balance the energy consumption among different sensors nodes. Finally Clustering is a main part in cooperative MIMO communications. Further investigations, for determining clusters, may include the use of other intelligent algorithms instead of GA.Other extension to proposed work is to study the impact of the different modulations on the network lifetime and might include cross layer optimization using routing techniques.

\section{REFERENCES}

[1] C. Shuguang, A. Goldsmith, "Energy-efficiency of MIMO and cooperative MIMO techniques in sensor networks," IEEE on Journal Selected Areas in Communications, vol. 22, no. 6, pp.1089--1098, Aug. 2004.

[2] W. B. Heinzelman, A. P. Chandrakasan, H. Balakrishnan, "An Application-Specific Algorithm Architecture for Wireless Microsensor Networks," IEEE Transactions on Wireless Communications, vol. 1, no. 4, pp. 660-670, 2002.

[3] O. Younis, M. Krunz, S. Ramasubramanian, "Node clustering in wireless sensor networks: recent developments and deployment challenges," IEEE Network - Special Issue on Wireless Sensor Networking 20 (3) (2006) 20-25.
[4] Liu, B., Wang, L., Jin, Y., “Advances in Differential Evolution," CHIN. J. Control Decision 2007, 22,721729.

[5] M. Sadeghian kerdabadi, R. GHazizadeh, R. sadeghian kerdabadi, "A novel clustering algorithm for wireless sensor networks based HBMO", Indian J.Sci.Res. 7 (1): 662-670, 2014.

[6] S. Hussain, O. Islam, A. Matin, "Genetic algorithm for energy efficient clusters in wireless sensor networks, " In Proceedings of the 4th International Conference on Information Technology, IEEE Computer Society, April 2007.

[7] J. Tillet, R. Rao, F. Sahin, "Cluster-head Identification in Ad Hoc Sensor Networks using Particle Swarm Optimization," IEEE International Conference on Personal Wireless Communications, December 2002, pp. 201-205.

[8] N.M.A. Latiff, C.C. Tsimenidis, B.S. Sharif, "Performance Comparison of Optimization Algorithm for Clustering in Wireless Sensor Networks," IEEE International Conference on Mobile Adhoc and Sensor Systems, pp.1-4 2007.

[9] I. Gupta, D. Riordan, S. Sampalli, "Cluster-head election using fuzzy logic for wireless sensor networks," Proceedings of Communication Networks and Services Research Conference (CNSR2), Halifax, Nova Scotia, Canada, pp. 255-260, 2005.

[10] Z.W. Siew, A. Kiring, H.T. Yew, P. Neelakantan K.T.K. Teo, "Energy Efficient Clustering Algorithm in Wireless Sensor Networks using Fuzzy Logic Control," Proc. 2011 IEEE Colloquium on Humanities, Science and Engineering Research (CHUSER 2011), pp. 392397, 2011.

[11] M. Afrashte Mehr, "Cluster Head Election using Imperialist competitive algorithm (CHEI) for wireless sensor networks," International Journal of Mobile Network Communications \& Telematics ( IJMNCT), Vol. 4, No.3, June 2014.

[12] M. L. D. Wong, A K. Nandi, "Automatic digital modulation recognition using artificial neural network and genetic algorithm. Signal Processing, 2004, 84(2), pp.351-365.

[13] L. Xiaohua, "Energy efficient wireless sensor networks with transmission diversity," IEEE Electronics Letters, vol.39, pp.1753-1755, Nov. 2003.

[14] D. jie, L. Dan-pu,W. Hua-ri, “ Energy efficiency of virtual MIMO transmission schemes forcluster-based wireless sensor networks," The Journal of China Universities of Posts and Telecommunications ,pp. 3138, August 2011.

[15] A. Duel-Hallen, "Decorrelating decision feedback multiuser detector for synchronous code-division multiple-access channel," IEEE Trans. Commun., vol. 41, no. 2, pp. 285-290, Feb. 1993.

[16] M. K. Varanasi, "Decision feedback multiuser detection: A systematic approach," IEEE Trans. Inform. Theory, vol. 45, no. 1, pp. 219-240, Jan. 1999.

[17] J. Xu, W. Su, M. Zhou, "Likelihood function-based modulation classification in bandwidth-constrained 
sensor networks. Proceedings of the 2010 IEEE International Conference on Networking, Sensing and Control (ICNSC'10), Apr 10-13, 2010, Chicago, IL, USA. Piscataway, NJ, USA: IEEE, 2010.

[18] N. Ahmadi, R.Berangi, "Modulation classification of QAM and PSK fromtheir constellation using genetic algorithm and hierarchical clustering," Proceedings of the International Conference on Information and Communication Technologies, From Theory to Applications (ICTTA'08), Apr 7-11, 2008, Damascus, Syria. Piscataway, NJ, USA: IEEE, 2008.

[19] V. K. Sachan, Syed A. Imam, M.T. Beg," Energyefficiency of Virtual Cooperative MIMO Techniques in Wireless Sensor Networks," International Conference on Computer Communication and Informatics (ICCCI2012), Jan. 2012.

[20] K. Xu, W. Yuan, W. Cheng, Y. Ding, Z. Yang," An Energy-efficient V-BLAST Based Cooperative MIMO Transmission Scheme for Wireless Sensor Networks",
IEEE Wireless Communications and Networking Conference,WCNC 2008.,pp. 688 - 693, April 2008.

[21] A. Afshara, O. Bozorg Haddada, M.A. Marin, B.J. Adamsd, "Honey-bee mating optimization (HBMO) algorithm for optimal reservoir operation," Journal of the Franklin Institute, 2007, pp. 452-462.

[22] V. K. Sachan, Syed A. Imam , M.T. Beg ," Energyefficiency of Virtual Cooperative MIMO Techniques in Wireless Sensor Networks," 2012 International Conference on Computer Communication and Informatics (ICCCI -2012), pp. 10 - 12, Jan, 2012.

[23] M. Krunz , M. Z. Siam , D. N. Nguyen," Clustering and power management for virtual MIMO communications in wireless sensor networks," Ad Hoc Networks journal, Volume 11, Issue 5, pp. 1571-1587, July 2013. 